\title{
Interpretation Method of GATEM Data Based on PID Controller Iteration Downward Continuation Method
}

\section{Shanshan Guan}

Jilin University

\section{Yu Zhu}

Jilin University

Bingxuan Du

Jilin University

Dongsheng Li

Jilin University

Yuan Wang

Jilin University

QIONG WU ( $\sim$ haideweidaowq@sina.cn )

Department of Instrumentation and Electrical Engineering, Jilin University, Changchun 130026, China https://orcid.org/0000-0003-3063-447X

\section{Yanju Ji}

Jilin University

\section{Full paper}

Keywords: Ground-source Airborne Time-domain Electromagnetic (GATEM), PID controller, mountainous zone

Posted Date: October 16th, 2020

DOl: https://doi.org/10.21203/rs.3.rs-92027/v1

License: (c) (i) This work is licensed under a Creative Commons Attribution 4.0 International License.

Read Full License 


\title{
Interpretation method of GATEM data based on PID controller iteration downward continuation method
}

Author \#1: Shanshan Guan, Department of Instrumentation and Electrical Engineering, Jilin

University, Changchun 130026, China, guanshanshan@jlu.edu.cn

Author \#2: Yu Zhu, Department of Instrumentation and Electrical Engineering, Jilin University, Changchun 130026, China, 1773856705@qq.com

Author \#3: Bingxuan Du, Department of Instrumentation and Electrical Engineering, Jilin University, Changchun 130026, China, haideweidaowq@sina.com

Author \#4: Dongsheng Li, Department of Instrumentation and Electrical Engineering, Jilin University, Changchun 130026, China, lidongsheng86@sina.cn

Author \#5: Yuan Wang, Department of Instrumentation and Electrical Engineering, Jilin University, Changchun 130026, China, wangyuan_ciee@jlu.edu.cn

Author \#6: Qiong Wu, Department of Instrumentation and Electrical Engineering, Jilin University, Changchun 130026, China, haideweidaowq@sina.cn

Author \#7: Yanju Ji, Department of Instrumentation and Electrical Engineering, Jilin University, Changchun 130026, China, 1183817666@qq.com

Corresponding author: Qiong Wu, Yanju Ji

\begin{abstract}
The Ground-source Airborne Time-domain Electromagnetic (GATEM) system has advantages for high efficiency and complex areas such as mountainous zone. The widely used section interpretation
\end{abstract}


method, ignoring the impact of flight height, which seriously affects the interpretation and imaging accuracy of shallow anomalies. The PID controller iteration downward continuation method is proposed. Based on the original continuation iteration method, the differential coefficient and integral coefficient are added. The result shows that the new method remarkably decreases the iteration number and the accuracy are verified by comparison with the numerical integration solution. The PID controller iteration downward continuation method is applied to the interpretation of GATEM data. For synthetic data, the after continuation interpretation results are closer to the true model than the $\mathrm{z}=30 \mathrm{~m}$ interpretation results. The method is also applied to GATEM field data in Yangquan City, Shanxi Province, China. The interpretation performs using PID controller iteration downward continuation results in a reliable GATEM field.

\section{Introduction}

The Ground-source Airborne Time-domain Electromagnetic (GATEM) system contains a long grounded electric source as the transmitter unit, and the portable receiving device is mounted on the platform of a rotor unmanned aerial vehicle (UAV) and airship as the aerial receiving devices. The GATEM system can realize efficient and fast geological survey tasks in complex areas, such as beach, volcanic geological structure, and seawater intrusion structure (Mogi, 2009; Ito et al., 2014; Yi et al., 2016; Li et al., 2019). It has the advantages of large exploration depth, low flight cost, and high security compared with those of the airborne time-domain electromagnetic system. In recent years, it has gradually become a research hotspot of the time-domain electromagnetic system.

To recognize a low resistivity anomaly, data procession is necessary for the GATEM field data. Scholars have carried out many studies on it. Sasaki et al. (2015) realized three-dimensional GREATEM inversion based on the least square method. This method is verified by a theoretical model; however, it is not used in field data interpretation. Abd Allah et al. (2016) applied a three-dimensional numerical simulation method by changing related parameters of the model to fit field data. Although the results are 
almost the same with geological data, it takes a lot of time. Liang et al. (2018) realized an iterative inversion algorithm for GREATEM using the deformed Born iteration method. This method was verified by the theoretical model and applied to the field data, and the results were basically consistent with the geological data. Wu et al. (2019) realized GATEM modeling and interpretation methods for a rough medium. The interpretation method based on artificial neural networks was applied to the field data, and the results were consistent with the geological data. A section interpretation method, the long wire source is solved by splitting into numbers of electric dipoles (Mogi et al., 1998; Ji et al., 2011), is widely used because of its simplicity and high efficiency (Mogi et al., 2009; Li et al., 2017, 2019; Ji et al., 2018). However, this method is limited to the high-precision interpretation of ground data. The effect of the flight altitude on interpretation is ignored during data processing, so the interpreted resistivity is bound to differ from the true resistivity. To avoid the impact of the flight altitude on interpretation, this paper proposes an interpretation method based on the downward continuation theory of the Proportional-Integral-differential (PID) controller iterative method.

The FFT downward continuation method has an amplification effect on high-frequency noise. To solve the problem, scholars have conducted relevant research in recent years. A stable downward continuation algorithm was presented (Fedi et al., 2002), which was based on the computation of stable vertical derivatives obtained by the ISVD method and Taylor series expansion of the potential field. Xu (2007) put forward the iteration method, which was much better than the FFT. At the same time, it did not require solving algebraic equations. Ali et al.(2018) presented a discrete equivalent source method to perform a stable downward continuation of gravity anomaly. Through automatically selecting an effective small amount of the discrete sources, the coefficient matrix size was reduced. The common methods have disadvantages in obtaining optimal results because of divergence and instability. The mean-value theorem for potential field was derived (Zhang et al., 2018). Compared with the fast Fourier transform Taylor series method and the integrated second vertical derivative Taylor series method, the method had very little boundary effect and was still stable in noise. The downward continuation method was also used to 
the aeromagnetic data (Cooper, 2016). By continuing the field to a level which was a fraction of the distance to the source, it ensured stability by preventing continuation down to or past the source, but its application was restricted to the tilt angle of vertically dipping vertically magnetized contacts. In order to solve the problems, it downward continued the data by a distance that was a fraction of the current depth, rather than by a fixed distance (Cooper, 2019). The new method was considerably more general in application.

When using the section interpretation method to interpret GATEM data, the accuracy of the shallow anomaly is affected by ignoring the flight altitude. In this paper, a PID controller iteration downward continuation method is proposed to improve the interpretation accuracy of GATEM data. In order to improve the calculation speed, the integral and differential terms are added to the original iteration formula, and the determination methods of the PID coefficients $k_{p}, k_{d}$ and $k_{i}$ are studied. The downward continuation theory is used in GATEM simulated data interpretation, and the results demonstrate that the method in this paper performs better than the traditional method. Finally, this method is also applied in GATEM field data of Yangquan, Shanxi Province, China.

\section{Methods}

The GATEM method mainly contains a several-km-long grounded electrical transmitter and an aircraft equipped with an induction coil and a receiver. The transmitter injects a bipolar square wave current into the ground. The vertical component of the magnetic field $(\mathrm{Hz})$ in a horizontal layered earth model is expressed as follows (Nabighian, 1988):

$$
H_{z}=\frac{I}{4 \pi} \int_{-L}^{L} \frac{y}{R} \int_{0}^{\infty}\left(1+r_{\mathrm{TE}}\right) e^{u_{0} z} \frac{\lambda^{2}}{u_{0}} \mathrm{~J}_{1}(\lambda R) d \lambda d x
$$

where $I$ is the transmitter current, $2 L$ is the length of the ground wire, $r_{\mathrm{TE}}$ is the reflection coefficient, $\lambda$ is the Hankel transform integral variable, $u_{0}=\lambda$ in the quasistatic electromagnetic field, $R=\left[\left(x-x^{\prime}\right)^{2}+y^{2}\right]^{1 / 2}$ 
case, $x^{\prime}$ is the horizontal longitudinal offset, $y$ is the horizontal transverse offset, and $z$ is the vertical offset, positive down.

Equation 1 can be calculated via the adoption of the Hankel transformation algorithm (Guptasarma and Singh, 1997). It can be converted from a frequency domain to a time domain by using the Guptasarma (1982) digital filtering method; then, a theoretical induced electromotive force at the $z=0$ plane is obtained. To verify the correctness of the algorithm, the result is compared with the analytical solution (Nabighian, 1984):

$$
\begin{aligned}
& \mathrm{v}=-\mu_{0} \mathrm{~s} \frac{\partial \mathrm{H}_{\mathrm{z}}}{\partial \mathrm{t}} \\
& =\frac{2 \mathrm{I}}{\pi \mu_{0} \sigma \mathrm{y}^{3}}\left\{\left(1+\theta^{2} \mathrm{y}^{2}\right) \mathrm{e}^{-\theta^{2} \mathrm{y}^{2}} \operatorname{erf}(\theta \mathrm{L})-\frac{\mathrm{L}}{\mathrm{R}}\left(1+\frac{\mathrm{y}^{2}}{2 \mathrm{R}^{2}}\right) \operatorname{erf}(\theta \mathrm{R})+\frac{\theta \mathrm{Ly}{ }^{2}}{\sqrt{\pi \mathrm{R}^{2}}} \mathrm{e}^{-\theta^{2} \mathrm{R}^{2}}\right\} \\
& \text { where } R=\left[y^{2}+L^{2}\right]^{1 / 2}, \quad \theta=\sqrt{\frac{\mu_{0} \sigma}{4 t}} \text {. }
\end{aligned}
$$

An example of a homogeneous half-space with $100 \Omega \cdot \mathrm{m}$ is calculated. The calculation parameters are as follows: the length of the long grounded electric source is $1 \mathrm{~m}$, the transmitter current $I=10 \mathrm{~A}$, the receiver coil equivalent area $S=1 \mathrm{~m}^{2}$, and the receiver location coordinates $x=0 \mathrm{~m}, y=400 \mathrm{~m}, z=0 \mathrm{~m}$. The calculated response is compared with the analytical solution (Equation 2), which is shown in Figure 1. They coincide with each other. The figure validates the correctness of the numerical simulation method.

Figure 2 shows a schematic diagram of measuring points in a 2D plane field. The electromagnetic responses at different measuring points are calculated by using the above method. Then, the theoretical electromagnetic responses of the observation surface $z=\mathrm{h}$ and the reference surface $z=0$ are constructed.

\section{The PID controller iteration downward continuation method}

Downward continuation theory

$\mathrm{Xu}$ (2007) proposed a downward continuation theory based on the iteration method. Its basic theory is that it transforms unstable downward continuation to stable upward continuation. First, the magnetic fields of the observation surface assign the reference surface as its initial values. Then, the magnetic fields 
at the reference surface are upward continued. Finally, the difference between the upward continuation result and the magnetic field at the observation surface is calculated, which is used to revise the magnetic fields at the reference surface. We repeat the process until precision is satisfied.

There is a difference between the electromagnetic response on the ground and in the air. The difference is larger at an earlier time. A continuation method of high precision is essential to improve data interpretation accuracy. When interpreted data are large, the continuation time will increase greatly, and the iterative process could not be completed. In this paper, the downward continuation that combines the PID controller theory is proposed. Based on the iteration method, a proportional-integral-differential is added. By adjusting the proportionality factor, differential coefficient, and integral coefficient together, the convergence speed is accelerated. The original iteration equation is as follows:

$$
u^{n+1}(x, y, z=0)=u^{n}(x, y, z=0)+k_{\mathrm{p}} \cdot e_{r}^{n}
$$

where $u^{n}(x, y, \mathrm{z}=0)$ is the electromagnetic response at the reference surface, $u^{n}(x, y, \mathrm{z}=h)$ is the upward continuation electromagnetic response at the observation surface, $\mathrm{n}$ is the number of iterations, and $e_{r}^{n}=u^{n}(x, y, z=h)-f(x, y, z=h)$ is an error term, $f(x, y, z=h)$ is the measured electromagnetic response at the observation surface. Equation (3) can be revised by the PID controller theory:

$$
u^{n+1}(x, y, z=0)=u^{n}(x, y, z=0)+k_{p} \cdot e_{r}^{n}+k_{i} \cdot \sum_{i=1}^{n} e_{r}^{i}+k_{d} \cdot\left(e_{r}^{n}-e_{r}^{n-1}\right)
$$

where $\sum_{i=1}^{n} e_{r}^{i}$ is the integral term and $e^{n}-e^{n-1}$ is the differentiation term.

Optimal parameters for the PID controller iteration downward continuation

Based on an original downward continuation iteration method, we add proportional-integral-differential coefficient by PID controller theory. The proportionality factor, differential coefficient, and integral coefficient can be determined by experience and the transient process curve. According to the empirical method, we first try the proportionality factor from large to small. When the transient process curve 
converges, we adjust the differential coefficient and the integral coefficient. Figure 3(a)-(c) show transient process curves that correspond to different PID parameters when $t=0.01 \mathrm{~ms}$. The number of iterations is 50000, 42710, and 31390. Figure 3(d)-(f) show transient process curves that correspond to different PID parameters when $t=0.25 \mathrm{~ms}$. The number of iterations is 4123,3415 , and 2094. Figure 3(g)-(i) show transient process curves that correspond to different PID parameters when $t=3.16 \mathrm{~ms}$. The number of iterations is 11040,8872 , and 5724 . Figure 3 shows that the number of iterations is different when the time is changing. The reason is that the absolute error varies between the electromagnetic response on the ground and in the air. The number of iterations is minimal when $k_{p}=4, k_{d}=0.9$ and $k_{i}=0.3$.

Figure 4 shows the transient process curves based on the original iteration and PID controller iteration when $t=0.05 \mathrm{~ms}$. The optimal proportionality factor of the original iteration is $k_{p}=2$. The parameters of the PID controller iteration are $k_{p}=4, k_{d}=0.9$ and $k_{i}=0.3$. The iteration numbers of both above are 16210 and 3155. These results indicate that the PID controller iteration downward continuation method is effective and efficient.

\section{Results}

\section{Precision validation}

To validate the effectiveness of the PID controller iteration downward continuation method, electromagnetic responses on the ground and in the air are calculated based on a uniform half-space model when the conductivity is equal to $0.01 \mathrm{~S} / \mathrm{m}$. The distance between the survey points is $10 \mathrm{~m}$. The survey points are selected randomly, which are at $x=0 \mathrm{~m}, y=150 \mathrm{~m} ; x=0 \mathrm{~m}, y=200 \mathrm{~m} ; x=150 \mathrm{~m}, y=$ $200 \mathrm{~m}$; and $x=200 \mathrm{~m}, y=200 \mathrm{~m}$. The PID controller iteration parameters are $k_{p}=4, k_{d}=0.9$ and $k_{i}=$ 0.3. The continued downward results are shown in Figure 5. The black line is the electromagnetic response for $\mathrm{h}=30 \mathrm{~m}$. The red dotted line is the analytical solution on the ground. The blue line is the electromagnetic response after downward continuation. The Figure 5 shows that continued downward results are coincide with analytical solution. The average relative error of all the survey points is only $0.08 \%$.

\section{Interpretation results}


Theoretical model

To verify the interpretation results of the downward continuation data, an example of a quasi-two-dimensional three-layer geology model was designed and is shown in Figure 6(a) and Figure 7(a). The calculation parameters are as follows: the start point of the transmitter line is $x=-500 \mathrm{~m}, y=0$ $\mathrm{m}$. The end point is $x=500 \mathrm{~m}, y=0 \mathrm{~m}$ on the ground. The transmitter current is $I=10 \mathrm{~A}$, the receiver coil equivalent area is $S=10000 \mathrm{~m}^{2}$, and the receiver location coordinates are $x=20 \mathrm{~m}-100 \mathrm{~m}, y=500$ $\mathrm{m}$ and $z=30 \mathrm{~m}$. The model parameters of Figure 6(a) are as follows: the bedrock has $200 \Omega \cdot \mathrm{m}$ resistivity, and the resistivity of the low-resistivity layer is recorded at $20 \Omega \cdot \mathrm{m}$. The depth of the low-resistivity layer varies from $-60 \mathrm{~m}$ to $-80 \mathrm{~m}$. The model parameters of Figure 7(a) are as follows: The depth of the low-resistivity layer varies from $-160 \mathrm{~m}$ to $-180 \mathrm{~m}$. The other model parameters are the same as in Figure 6(a). The $z=30 \mathrm{~m}$ electromagnetic responses are calculated, which are continuated downward to the ground. Then the after continuation electromagnetic response and $z=30 \mathrm{~m}$ electromagnetic response are interpreted (Ji et al., 2011). The long wire source is solved by splitting into numbers of electric dipoles The electromagnetic response of wire source can be:

$$
\mathrm{V}_{z}=\sum_{i=1}^{N} \frac{I S \mu y d s}{8 \pi t \theta^{2} r_{i}^{5}}\left[3 \operatorname{erf}\left(\theta r_{i}\right)-\frac{2}{\sqrt{\pi}} \theta r_{i}\left(3+2 \theta^{2} r_{i}^{2}\right) e^{-\theta^{2} r_{i}^{2}}\right]
$$

where $I$ is the transmitter current, $S$ is the area of coil sensor, $\mu$ is the magnetic permeability, $d s$ is the length of electric dipole, $t$ is the sampling time, $r_{i}=\sqrt{\left(x-x_{i}\right)^{2}+y^{2}}, x$ is $x$ coordinates of the observation point, $y$ is $y$ coordinates of the observation point, $\theta=\sqrt{\frac{\mu}{4 t \rho}}, \rho$ is the resistivity. After the value of $\theta$ is obtained, the apparent resistivity will be $\rho=\frac{\mu}{4 t \theta^{2}}$. The interpretation results are shown in Figure 6(b), (c) and Figure 7(b), (c).

Figures 6(b) and 7(b) are the interpretation results of the $z=30 \mathrm{~m}$ electromagnetic response, and Figures 6(c) and 7(c) are the interpretation results of after continuation electromagnetic response. For the shallow abnormality case, the results of the $z=30 \mathrm{~m}$ electromagnetic response, as shown in Figure 6(b), indicate that the thickness is much greater than that of the results for after continuation electromagnetic 
response, as shown in Figure 6(c). For the deep abnormality case, the results of the low resistivity abnormality are similar. But the shallow part of after continuation electromagnetic response, as shown in Figure 7(c), is more similar to the true model than the results of the $z=30 \mathrm{~m}$ electromagnetic response, as shown in Figure 7(b). The results show that after continuation electromagnetic response are nearly equivalent to the true model. The accuracy of the data interpretation could be improved by PID controller iteration downward continuation method.

Field data results

To verify the effectiveness of the PID controller iteration downward continuation method, it is applied to the field data. We conducted an exploration experiment at Yangquan City, Shanxi Province, China, in 2016 December by using a GATEM system. The field site in Yangquan City was chosen because there is a borehole in the survey area. According to the Shanxi Geological Survey, the order of the geological structures from the top to the bottom is Quaternary strata, Permian system, Carboniferous system and Ordovician system. The Quaternary strata mainly consists of loess and clay, and the resistivity is relatively low. The Permian system is composed of mudstone, sandstone, fine-grained sandstone and coal seams, and the resistivity is relatively high. It is the main coal-bearing stratum that can be exploited. The main constituents of the Carboniferous system are siltstone, mudstone, bauxite, limestone and coal seam. The Ordovician system is the basement of the coal seam, and the main lithology is dark gray layered limestone with high resistivity. The survey area covers the known mined-out area. The resistivity of this area is very low, and the depth of the surface is approximately $100 \mathrm{~m}$. The thickness of the mined-out area is $20 \mathrm{~m}$. The mined-out area and the Permian system contain water; therefore, the resistivity may be lower than itself, and the low-resistivity layer may be thicker than the mined-out area thickness.

The survey area and flight paths are shown in Figure 8. The flight path is parallel to the long wire source line. The electrical transmitting system used a grounded wire source with a length of $2 \mathrm{~km}$. The transmitter current was $40 \mathrm{~A}$, the frequency was $12.5 \mathrm{~Hz}$, and the transmitter waveform was a bipolar square wave. The equivalent area of the receiving coil was $2,160 \mathrm{~m}^{2}$, the sampling rate of the receiving system was $30 \mathrm{kHz}$, the receiver location coordinates were $x=60 \mathrm{~m}-1980 \mathrm{~m}, y=300 \mathrm{~m}$ and the flight altitude was $z=30 \mathrm{~m}$. 
Baseline correction was performed on flight data by a method based on wavelet transform (Wang et al., 2013). Decay curves were stacked 8 times to suppress random noise. To remove the electromagnetic signal noise, an exponential fitting-adaptive Kalman filter (EF-AKF) was used (Ji et al., 2018). The denoised field data was continuation downward to the ground. The data of after downward continuation, the results of raw data and the denoised $z=30 \mathrm{~m}$ field data were interpreted (Ji et al., 2011). The depth section images are shown in Figure 9.

Figure 9(a) presents the interpretation results of raw data, Figure 9(b) presents the results of the denoised $z=30 \mathrm{~m}$ field data, Figure 9(c) presents the results of the data after downward continuation. The low resistivity layer under $200 \mathrm{~m}$, in Figure 9(a), is the mined-out area with accumulated water, which is much thicker than its true thickness. The low resistivity layer at $100 \mathrm{~m}$, in Figure 9(b) and (c), which are consistent with the survey area. The results of the $z=30 \mathrm{~m}$ field data, as shown in Figure 9(b), indicate that the thickness of the mined-out area is greater than that of the results for data after downward continuation, as shown in Figure 9(c). The results show that the PID controller iteration downward continuation method can be effectively applied to the denoised field data and it can approve the interpretaion accuracy of GATEM data.

\section{Conclusion}

The GATEM system is an effective method for the exploration of low resistivity anomalies in complex areas. In this paper, a PID controller iteration downward continuation method is proposed to improve the interpretation and imaging accuracy of data. The integral and differential term are added into the traditional iterative method based on the PID controller theory. Compared with the analytical solution, the continuation accuracy of the iterative method is verified, and the iteration time is effectively reduced. This method is validated using synthetic data interpretation and is compared with the interpretation results of the $z=30 \mathrm{~m}$ electromagnetic response. The results show that interpretation of after continuation electromagnetic response is more consistent with the true model, especially for the shallow anomalous body. To further verify the effectiveness, this method is applied to field data of Yangquan City, Shanxi Province, China, which improves the quality of the GATEM field data results. This method has significant implications for the GATEM exploration.

\section{Declaration}


Availability of data and materials

The data are available by sending email to corresponding authors.

Competing interests

No competing interests.

Funding

'3D Numerical Simulation of ATEM Based on Conformal Mesh Technique (JJKH20190127KJ)' supported by the Planning Project of Science and Technology and Humanities and Social Science Research of the Education Department of Jilin Province, 'Research on meshless implicit finite difference three-dimensional numerical simulation of the ATEM exploration (41604084,41674109)' supported by the National Natural Science Foundation of China.

Authors' contributions

Shanshan Guan: She pointed out the overall research ideas of the article and provided technical support.

Yu Zhu: He provided technical support.

Bingxuan Du: He modified articles.

Dongsheng Li: He provided GATEM synthetic data.

Yuan Wang: He provided GATEM field data.

Qiong Wu: She provided technical support of section interpretation method and interpreted GATEM data.

Yanju Ji: She provided technical support.

\section{Acknowledgments}

This study was carried out within the framework of the project '3D Numerical Simulation of ATEM Based on Conformal Mesh Technique (JJKH20190127KJ)' supported by the Planning Project of Science and Technology and Humanities and Social Science Research of the Education Department of Jilin 
Province and the project 'Research on meshless implicit finite difference three-dimensional numerical simulation of the ATEM exploration (41604084,41674109)' supported by the National Natural Science Foundation of China. We thank the anonymous reviewers and editors for their helpful comments.

\section{References}

Abd Allah S, Mogi T (2016) Three-dimensional resistivity modeling of GREATEM survey data from ontake volcano, northwest Japan[J]. Earth Planets \& Space, 68(1): 76.

https://doi.org/10.1186/s40623-016-0443-Z

Ali, A.E.O.A. 1 ; Zhan Liu 1 ; Yongliang Bai 1 ; Farwa, A.G. 2 ; Ahmed, A.S. 3 ; Guomin Peng 1 (2018) A stable gravity downward continuation for structural delineation in Sulu Sea region, Journal of Applied Geophysics, 155, 26-35. https://doi.org/10.1016/j.jappgeo.2018.05.009

Cooper, G. R. J (2016) The downward continuation of the Tilt-Angle, Near Surface Geophysics, 14(5), 385-390. https://doi.org/10.3997/1873-0604.2016022

Cooper, G. R. J (2019) The downward continuation of aeromagnetic data from magnetic source ensembles, Near Surface Geophysics, 17, 101-107. https://doi.org/10.1002/nsg.12035

Fedi. M and Florio.G (2002) A stable downward continuation by using the ISVD method, Geophysics Journal International, 151, 146-156. https://doi.org/10.1046/j.1365-246X.2002.01767.x

Guptasarma, D (1982) Optimization of short digital linear filters for increased accuracy, Geophysical Prospecting, 30, 501-514. https://doi.org/10.1111/j.1365-2478.1982.tb01320.x

Guptasarma, D., Singh, B (1997) New digital linear filters for Hankel J0 and J1 transforms, Geophysical Prospecting, 45, 745-762. https://doi.org/10.1046/j.1365-2478.1997.500292.x

Ito, H., Kaieda, H., Mogi, T., Jomori, A., and Yuuki, Y (2014) Grounded electrical-source airborne transient electromagnetics (GREATEM) survey of Aso Volcano, Japan, Explor. Geophys., 45, 43-48. https://doi.org/10.1071/EG12074

Ji, Y., Yang, G., Guan, S (2011) Interpretation Research on Electrical Source of Time Domain Ground -airborne Electromagnetic data. 2011 International Conference on Green Environmental Sustainable Development.

Ji, YJ (Ji, Yanju) 1,2, Wu, Q (Wu, Qiong) 1, Wang, Y (Wang, Yuan) 1, Lin, J (Lin, Jun) 1,2 , Li, DS (Li, Dongsheng) 1, Du, SY (Du, Shangyu) 1 , Yu, SB (Yu, Shengbao) 1, Guan, SS (Guan, Shanshan) 1(2018) 
Noise reduction of grounded electrical source airborne transient electromagnetic data using an exponential fitting-adaptive Kalman filter, Exploration Geophysics, 49(3), 243-252.

https://doi.org/10.1071/EG16046

Li, D. a ,c , Wang, Y. a ,c , Lin, J. a ,b ,c , Yu, S. a ,b , Ji, Y. a ,b (2017) Electromagnetic noise reduction in grounded electrical-source airborne transient electromagnetic signal using a stationary wavelet- based denoising algorithm. Near Surface Geophysics, 15(2), 163-173.

https://doi.org/10.3997/1873-0604.2017003

Li, D., Tian, Z., Ma, Y., Gu, J., Ji, Y., Li, S (2019) Application of Grounded Electrical Source Airborne Transient Electromagnetic (GREATEM) System in Goaf Water Detection, Journal of environmental \& engineering geophysics, 24(3), 387-397. https://doi.org/10.2113/JEEG24.3.387

Bingyang Liang 1, Chen Qiu 1, Feng Han 1, Chunhui Zhu 1, Na Liu 1, Hai Liu 1, Fubo Liu 2 , Guangyou Fang 2 , Qing Huo Liu 3 (2018) A New Inversion Method Based on Distorted Born Iterative Method for Grounded Electrical Source Airborne Transient Electromagnetics. IEEE Transactions on Geoscience and Remote Sensing, 56(2), 877-887. https://doi.org/10.1109/TGRS.2017.2756086

Nabighian, M (1984) Foreword and introduction: Geophsics, 49, 849-853.

Nabighian, M (1988) Electromagnetic methods in applied geophysics, Vol. 1, Theory. Society of Exploration Geophysicists, Tulsa, OK,528.

Mogi, T., Tanaka, Y., Kusunoki, K., Morikawa, T., and Jomori, N (1998) Development of Grounded Electrical Source Airborne Transient EM(GREATEM), Exploration Geophysics, 29, 61-64. https://doi.org/0812-3985;1834-7533

Toru Mogi; Ken'ichirou Kusunoki; Hideshi Kaieda; Hisatoshi Ito; Akira Jomori; Nobuhide Jomori and Youichi Yuuki (2009) Grounded electrical-source airborne transient electromagnetic (GREATEM) survey of Mount Bandai, north-eastern Japan. Exploration Geophysics, 40(1), 1-7.

https://doi.org/10.1071/EG08115

Yutaka Sasaki a, Myeong-Jong Yi b, Jihyang Choi b , Jeong-Sul Son b (2015) Frequency and time domain three-dimensional inversion of electromagnetic data for a grounded-wire source. Journal of Applied Geophysics, 112, 106-114. https://doi.org/10.1016/j.jappgeo.2014.09.016

Wang, Y, Ji, Y., Li, S., Lin, J., Zhou, F., and Yang, G (2013) A wavelet-based baseline drift correction method for grounded electrical source airborne transient electromagnetic signals, Explor. Geophys., 44, 229-237. https://doi.org/10.1071/EG12078 
Wu, Q 1, Li, D S 1 , Jiang, C D 1 , Ji, Y J 2 , Wen, Y L 1, Luan, H 1 (2019) Ground-source Airborne Time-domain ElectroMagnetic (GATEM) modelling and interpretation method for a rough medium based on fractional diffusion. Geophysical Journal International, 217(3),1915-1928.

https://doi.org/10.1093/gji/ggz128

$\mathrm{Xu}, \mathrm{S}$ (2007) A comparison of effects between the iteration method and FFT for downward continuation of potential fields. Chinese Journal of Geophysics, 50(1), 285-289. https://doi.org/10.1002/cjg2.1032

Yi, M.J., Kim, J.H., Sung, N.H., Han, M.H., Motschka, K., Supper, R., Ahl, A., and Johmori, A (2016) Test Airborne EM Surveys at a Black-shale Type Uranium Deposit in Korea, Near Surface Geoscience 2016 - 22nd European Meeting of Environmental and Engineering Geophysics.

https://doi.org/10.3997/2214-4609.201601940

Chong Zhang, Qingtian Lu, Jiayong Yan, Guang Qi (2018) Numerical Solutions of the Mean-Value Theorem: New Methods for Downward Continuation of Potential Fields. Geophysical Research Letters, 3461-3470. https://doi.org/10.1002/2018GL076995

Figure 1. Comparison of the numerical simulation and analytical solution of the GATEM response in a homogeneous half-space.

Figure 2. Schematic diagram of measuring points in a two-dimensional (2D) plane field.

Figure 3. PID transient process curves.(a)-(c) transient process curves that correspond to different PID parameters when $t=0.01 \mathrm{~ms}$. The number of iterations is 50000, 42710, and 31390. (d)-(f) transient process curves that correspond to different PID parameters when $t=0.25 \mathrm{~ms}$. The number of iterations is 4123, 3415, and 2094. (g)-(i) transient process curves that correspond to different PID parameters when $t$ $=3.16 \mathrm{~ms}$.

Figure 4. Transient process curves based on the original iteration and PID controller iteration when $t=$ $0.05 \mathrm{~ms}$. 
Figure 5. Downward continuation results at different survey points (a) $x=0 \mathrm{~m}, y=150 \mathrm{~m}$ (b) $x=0 \mathrm{~m}, y$ $=200 \mathrm{~m}$ (c) $x=150 \mathrm{~m}, y=200 \mathrm{~m}$ and (d) $x=200 \mathrm{~m}, y=200 \mathrm{~m}$.

Figure 6. Comparison between depth section images of the shallow abnormality: (a) theoretical model, (b) interpretation results derived from the $z=30 \mathrm{~m}$ electromagnetic response, and (c) interpretation results derived from downward continuation of $z=30 \mathrm{~m}$ electromagnetic response. The purple outlines serve as a reference to the theoretical model.

Figure 7. Comparison between depth section images of the deep abnormality: (a) theoretical model, (b) interpretation results derived from the $z=30 \mathrm{~m}$ electromagnetic response, and (c) interpretation results derived from downward continuation of $z=30 \mathrm{~m}$ electromagnetic response. The purple outlines serve as a reference to the theoretical model.

Figure 8. Survey area for Yangquan, Shanxi Province in China. The red line is the dipole source location, which has a length of $2 \mathrm{~km}$, and the electrical dipole $\mathrm{A}$ is 0 . The yellow line is the flight path which is parallel to the red line. The blue line is the chosen section for verifying the application of the downward continuation.

Figure 9. Depth section images from the field data. (a) the results of raw data, (b) the results of the $z=30$ $\mathrm{m}$ field data, (c) the interpretation results of the data after downward continuation. 
Figures

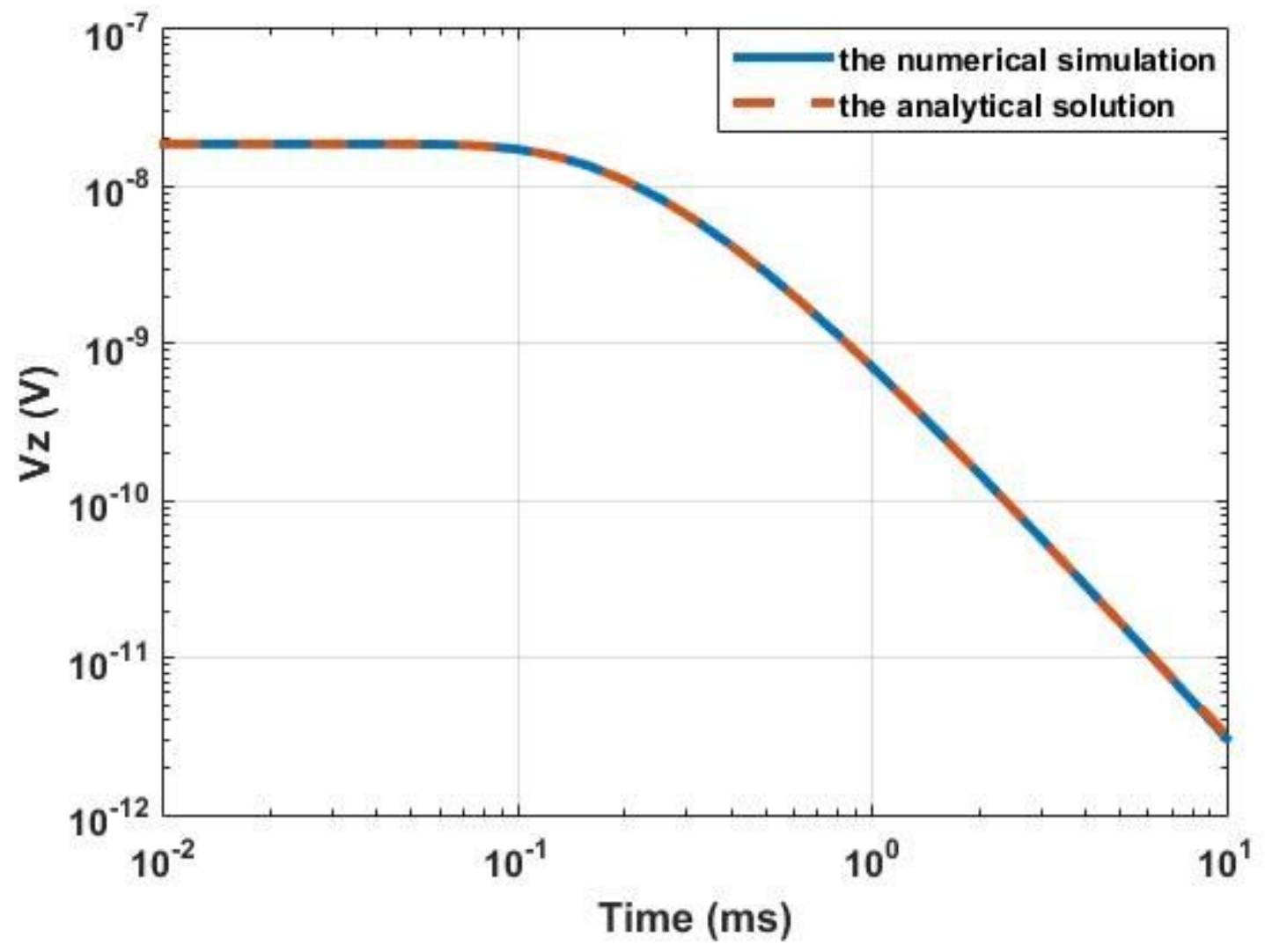

Figure 1

Comparison of the numerical simulation and analytical solution of the GATEM response in a homogeneous half-space. 

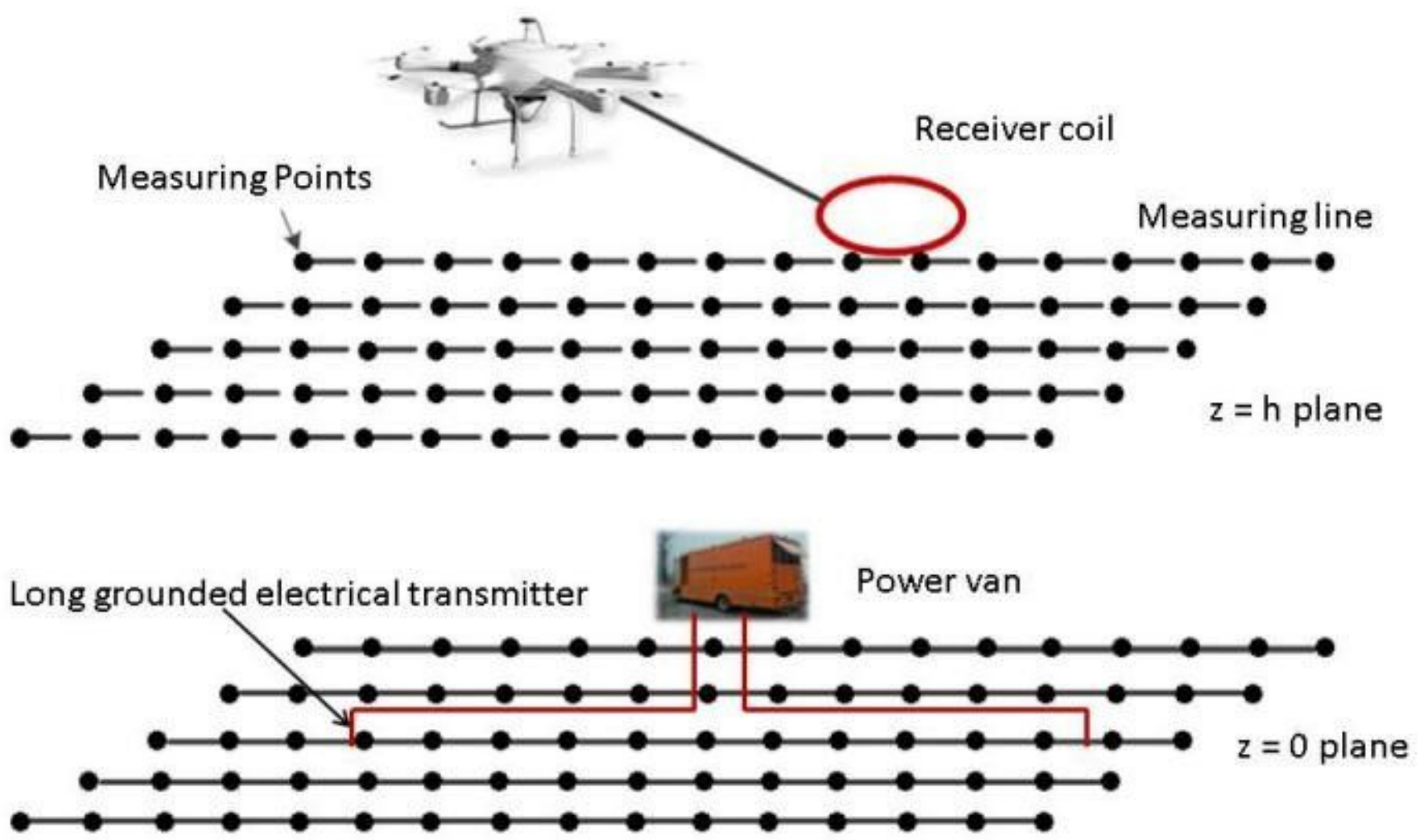

Figure 2

Schematic diagram of measuring points in a two-dimensional (2D) plane field. 

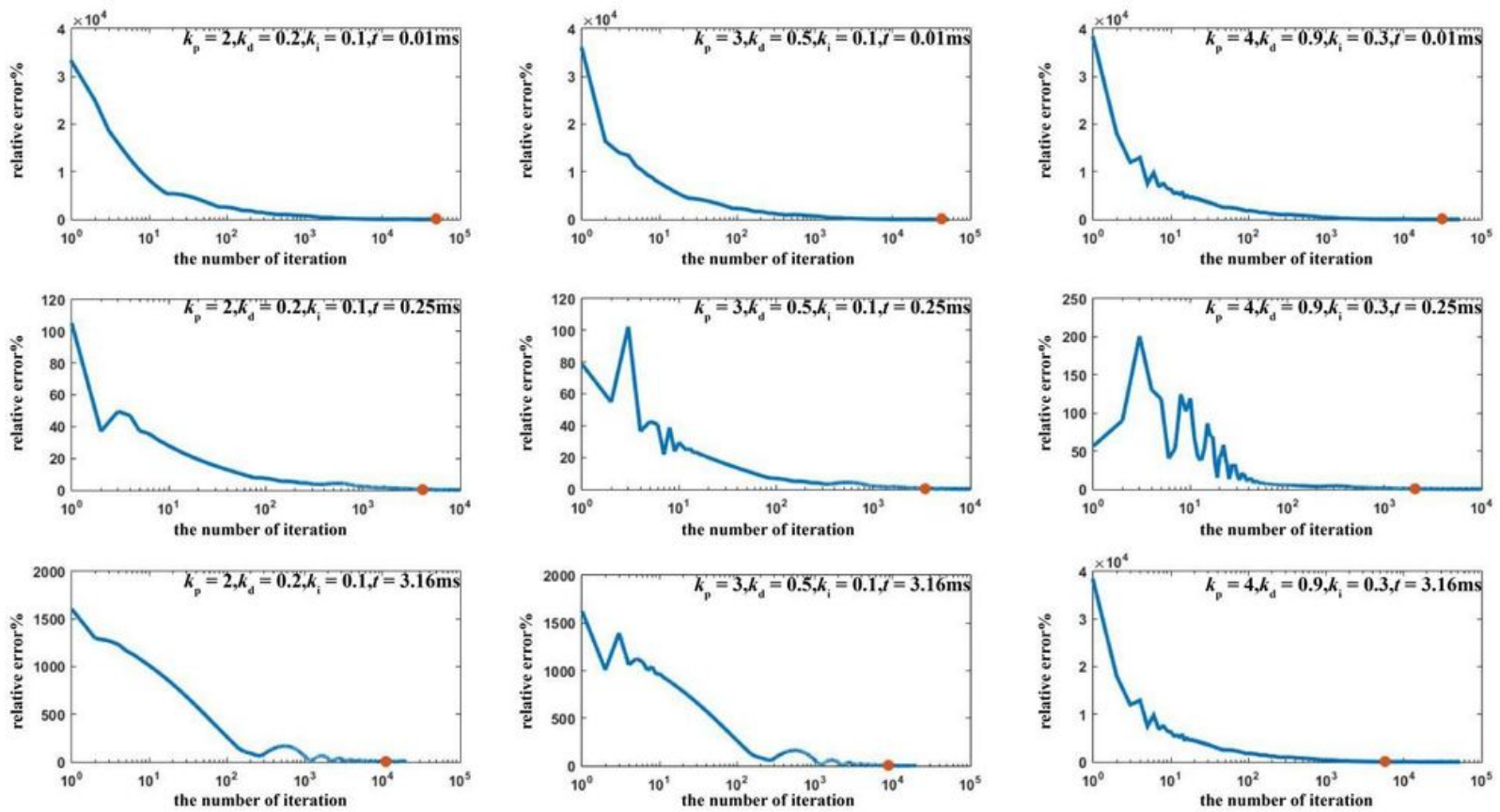

\section{Figure 3}

PID transient process curves.(a)-(c) transient process curves that correspond to different PID parameters when $t=0.01 \mathrm{~ms}$. The number of iterations is 50000,42710 , and 31390. (d)-(f) transient process curves that correspond to different PID parameters when $t=0.25 \mathrm{~ms}$. The number of iterations is 4123,3415 , and 2094. (g)-(i) transient process curves that correspond to different PID parameters when $\mathrm{t}=3.16 \mathrm{~ms}$. 


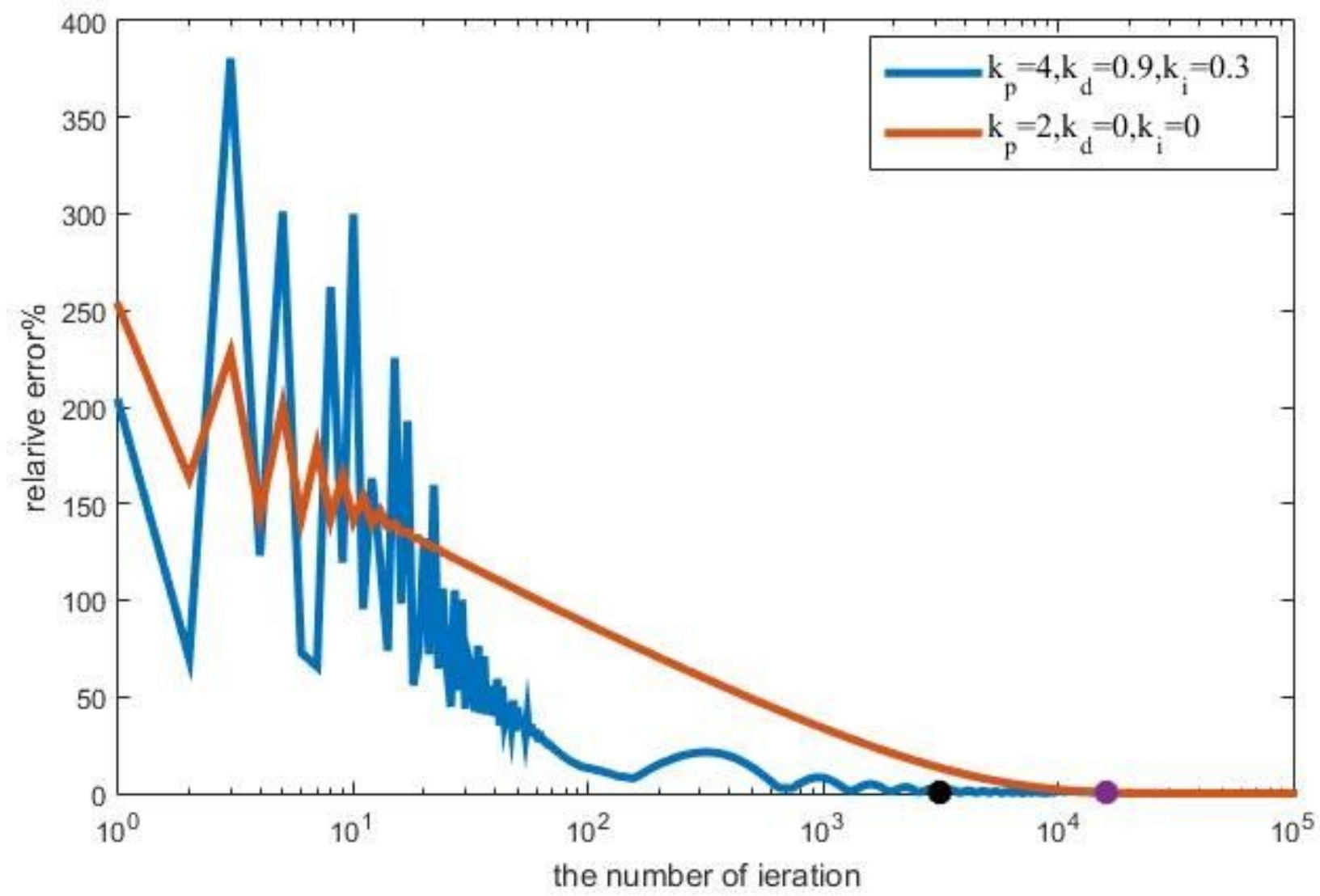

Figure 4

Transient process curves based on the original iteration and PID controller iteration when $t=0.05 \mathrm{~ms}$.
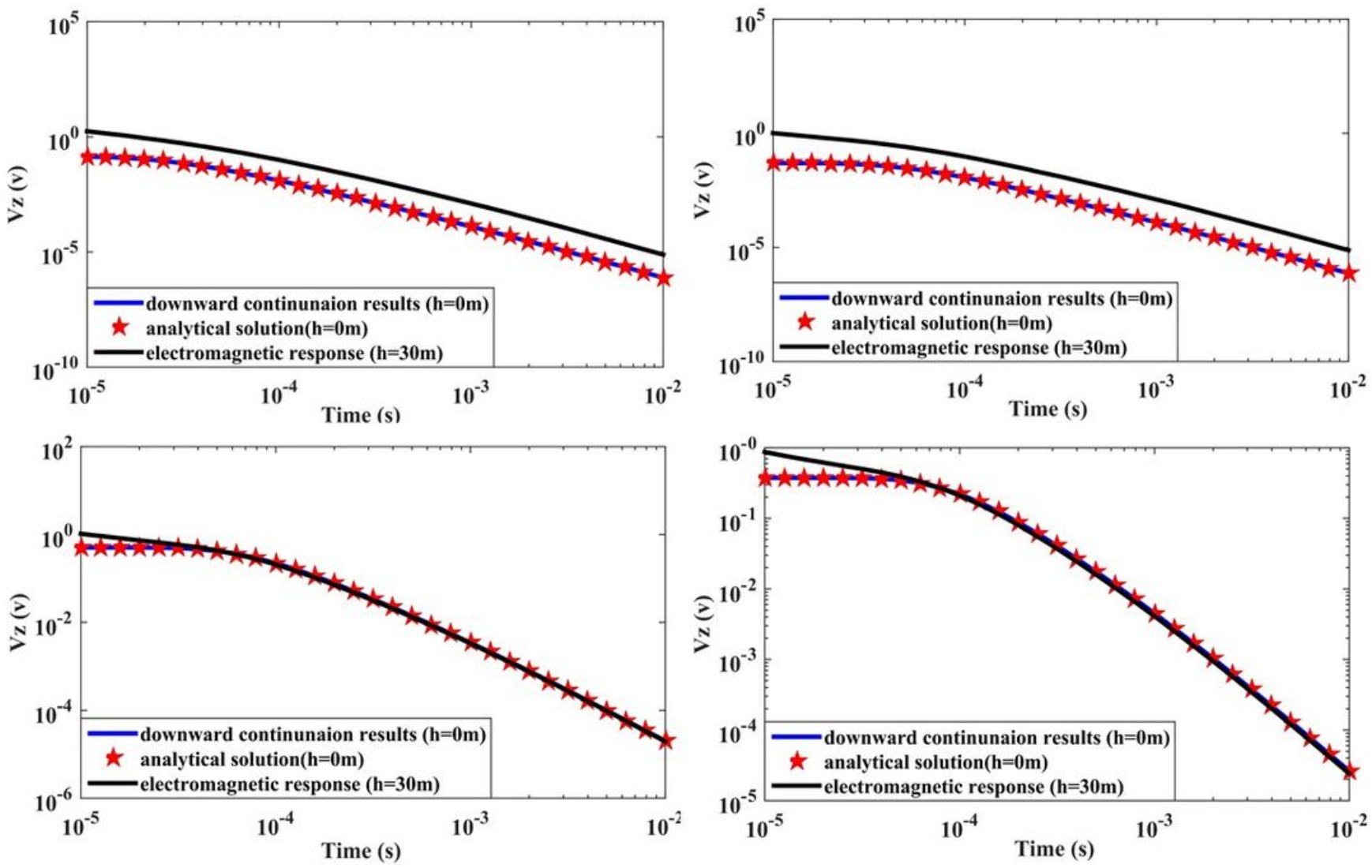


\section{Figure 5}

Downward continuation results at different survey points (a) $x=0 \mathrm{~m}, y=150 \mathrm{~m}$ (b) $x=0 \mathrm{~m}, \mathrm{y}=200 \mathrm{~m}$ (c) $x=150 m, y=200 m$ and $(d) x=200 m, y=200 m$.

(a)

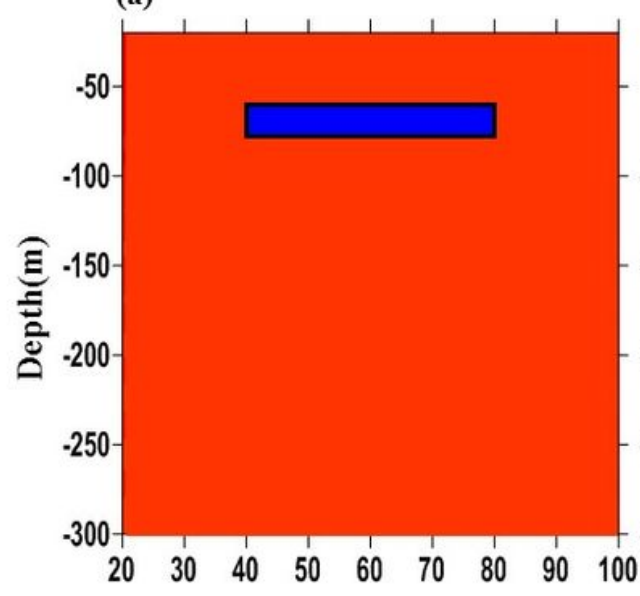

(b)

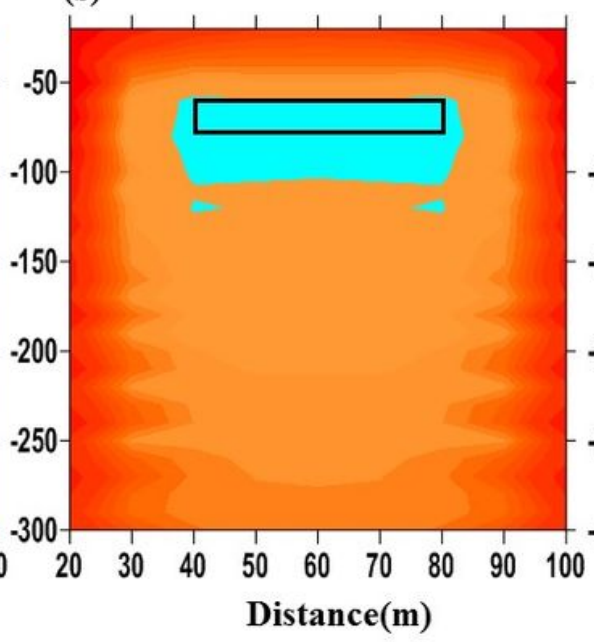

(c)

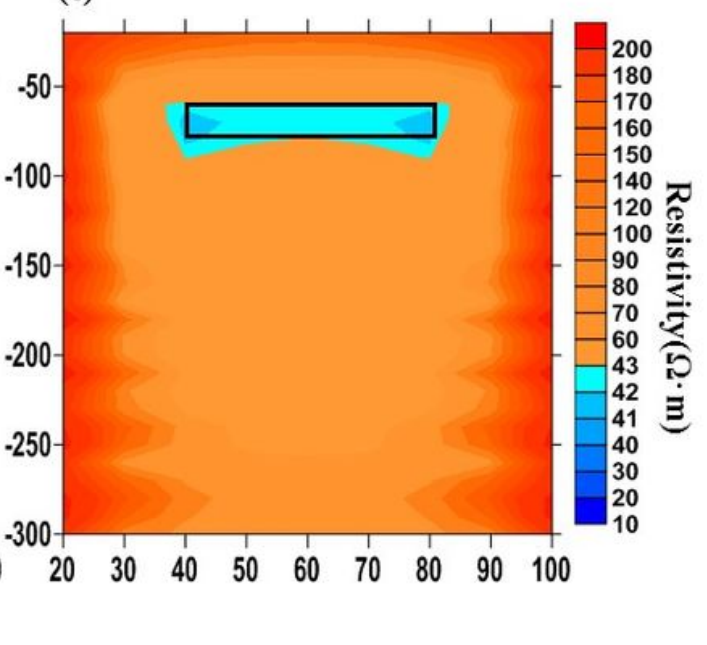

Figure 6

Comparison between depth section images of the shallow abnormality: (a) theoretical model, (b) interpretation results derived from the $z=30 \mathrm{~m}$ electromagnetic response, and (c) interpretation results derived from downward continuation of $z=30 \mathrm{~m}$ electromagnetic response. The purple outlines serve as a reference to the theoretical model. 
(a)

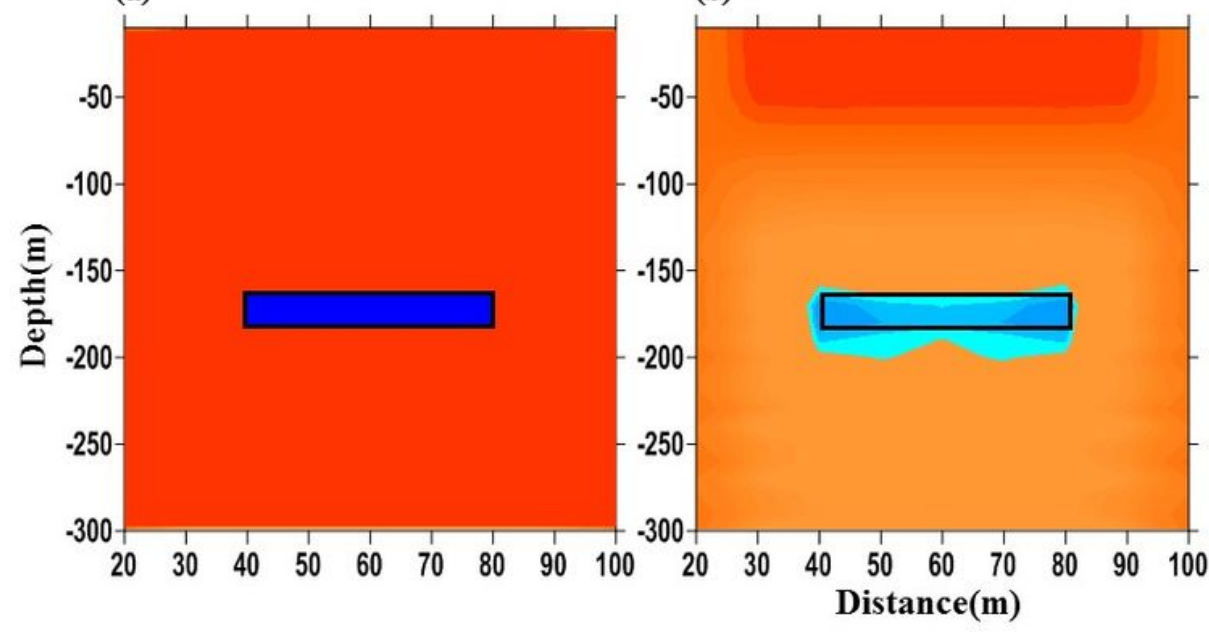

(c)

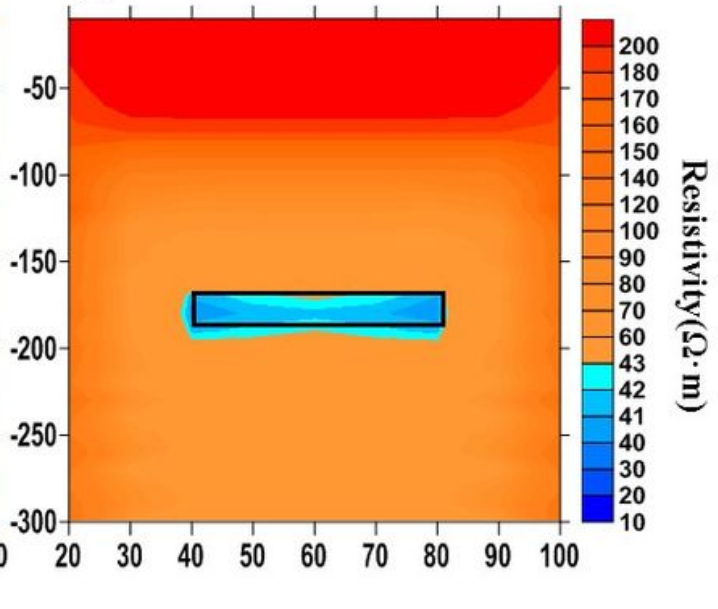

\section{Figure 7}

Comparison between depth section images of the deep abnormality: (a) theoretical model, (b) interpretation results derived from the $\mathrm{z}=30 \mathrm{~m}$ electromagnetic response, and (c) interpretation results derived from downward continuation of $z=30 \mathrm{~m}$ electromagnetic response. The purple outlines serve as a reference to the theoretical model. 


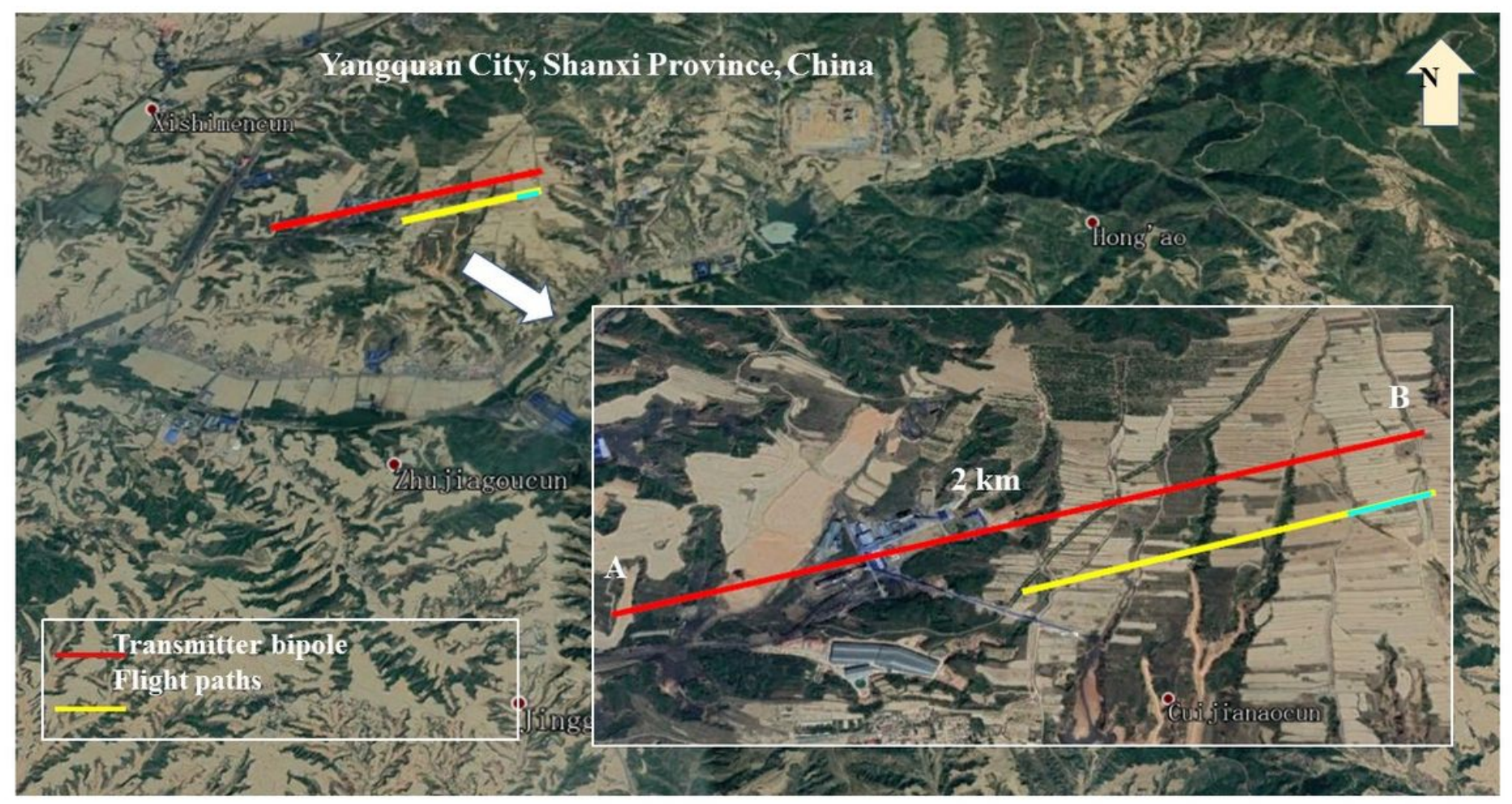

\section{Figure 8}

Survey area for Yangquan, Shanxi Province in China. The red line is the dipole source location, which has a length of $2 \mathrm{~km}$, and the electrical dipole $A$ is 0 . The yellow line is the flight path which is parallel to the red line. The blue line is the chosen section for verifying the application of the downward continuation. Note: The designations employed and the presentation of the material on this map do not imply the expression of any opinion whatsoever on the part of Research Square concerning the legal status of any country, territory, city or area or of its authorities, or concerning the delimitation of its frontiers or boundaries. This map has been provided by the authors. 

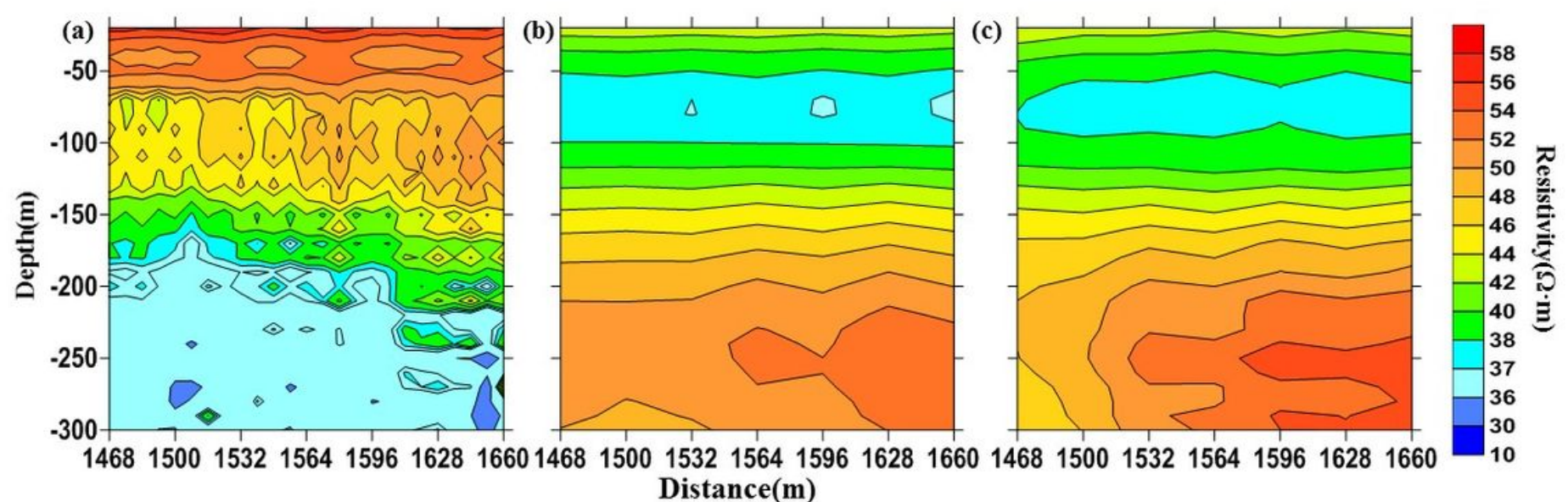

\section{Figure 9}

Depth section images from the field data. (a) the results of raw data, (b) the results of the $z=30 \mathrm{~m}$ field data, (c) the interpretation results of the data after downward continuation.

\section{Supplementary Files}

This is a list of supplementary files associated with this preprint. Click to download.

- Graphicalabstract.png 